\title{
A novel long non-coding RNA GK-IT1 promotes the carcinogenesis and progression of esophageal squamous cell carcinoma via mediating the phosphorylation of MAPK1
}

\section{Xin Yang}

The First Affiliated Hospital of Chongqing Medical University

\section{Tian Yang Zeng}

The First Affiliated Hospital of Chongqing Medical University

\section{Zi Yang Liu}

The First Affiliated Hospital of Chongqing Medical University

\section{Wan Lun He}

The First People's Hospital of Chongqing Liangjiang New Area

\section{Meng Ting Hu}

Chongqing Medical University

\section{Ti Tang}

The First Affiliated Hospital of Chongqing Medical University

\section{Lei Xing}

The first affiliated Hospital of Chongqing Medical University

\section{Li Chen ( $D$ Drchenli@sina.com )}

The First Affiliated Hospital of Chongqing Medical University https://orcid.org/0000-0002-7739-3741

\section{Research}

Keywords: Long non-coding RNAs, GK-IT1, MAPK1, phosphorylation, esophageal squamous cell carcinoma,

Posted Date: December 23rd, 2020

DOl: https://doi.org/10.21203/rs.3.rs-131952/v1

License: (1) This work is licensed under a Creative Commons Attribution 4.0 International License. Read Full License 


\section{Abstract}

Background: Recent studies have shown that Long non-coding RNAs (IncRNAs) are crucial in the invasion, angiogenesis, progression, and metastasis of esophageal squamous cell carcinoma (ESCC). However, the biological functions and potential molecular mechanism of LncRNA GK-IT1 in esophageal squamous cell carcinoma has not been reported.

Methods: We analysed the expression of GK-IT1 in ESCC and their adjacent normal tissues in the TCGA database. The quantitative real-time-PCR (qRT-PCR) was used to detect the expression of GK-IT1 in Clinical specimens. The Kaplan-Meier method was employed to draw the survival curve and then the statistical significance was calculated using the logarithmic rank test. a range of functional experiments in vivo and in vitro were used to explore the role of GK-IT1 in the carcinogenesis and development of ESCC. RNA pull down assay, RNA immunoprecipitation (RIP), fluorescence in situ hybridisation (FISH), agarose gel electrophoresis and immunofluorescence were all employed to explore the interaction mechanism between GK-IT1 and MAPK1 (mitogen activated protein kinase 1).

Results: The expression of GK-IT1 was higher in ESCC than adjacent normal tissues, which was positively correlated with the clinical stage and shorter survival time. The knockout of the GK-IT1 gene significantly attenuated the abilities of ESCC cell proliferation, invasion and migration, induced apoptosis and autophagy in ESCC cells and inhibited tumour growth and tumour metastasis in vivo. on the contrary, the upregulation of GK-IT1 had the opposite effect. Further studies have shown that GK-IT1 can regulate the biological process of ESCC by regulating the phosphorylation of MAPK1.

Conclusion: Our study reveals that GK-IT1 mediated the phosphorylation of MAPK1 improve the carcinogenesis and development of esophageal squamous cell carcinoma through ERK/MAPK pathway which indicates that GK-IT1 possesses substantial potential as a novel biomarker for ESCC prognosis and therapy.

\section{Background}

Esophageal cancer (ESCA) is one of the most aggressive malignant tumours, ranking sixth for morbidity and mortality in the world [1]. Being among the most aggressive human cancers worldwide and the second most common cancer. In China, however, esophageal squamous cell carcinoma (ESCC) is classed as a major histological type of esophageal cancer based on pathological classifications [2]. Current epidemiological studies mostly focus on the external environmental factors that may play a role in the carcinogenesis of esophageal cancer, such as smoking and drinking. However, it was rarely studied regarding the contribution of internal molecular and genetic factors to the promotion of esophageal cancer. Surgery, radiotherapy and chemotherapy have improved the possibilities for recovery, but the overall survival rate for patients with esophageal cancer is still low and the treatment cost is high. For these reasons, finding feasible molecular targets for the treatment of esophageal cancer is urgently needed $[3,4]$. 
Eighty percent of the human genome is transcribed into RNA, whereas only approximately $1.2-2 \%$ $(\sim 20,000)$ of the total human genome sequence is encoded with protein-coding genes. The majority of the human genome is transcribed into non-protein-coding RNAs (ncRNAs). Not only that, the dysregulation of IncRNAs has been demonstrated to an important factor in the occurrence and development of numerous diseases and human cancers [5]. IncRNAs are participated in a multilevel regulation of gene expression, including transcriptional regulation by recruitment of chromatin-modifying complexes [6] and post-transcriptional regulation by interaction with miRNAs, mRNAs, or proteins [7]. Furthermore, they can regulate many of cancer's characteristics, including proliferation [8], apoptosis [9], metastasis [10] , metabolism [11] and other biological processes.

LncRNAs have also been regarded as potential biomarkers and therapeutic targets for cancers. For instance, Shan et al. found that through regulation in the cell cycle and endogenous apoptosis, LINC00908 can have important proliferation and anti-apoptotic effects in colorectal cancer. Due to this, LINC00908 may become a potential biomarker and new type of therapy for colorectal cancer [12]. Han et al. demonstrated that the upregulation of ZNF649-AS1 expression induced by H3K27ac modification may lead to autophagy and trastuzumab resistance by binding to PTBP1 and promoting ATG5 transcription [13]. In addition, it has also been reported that LncHERES is a trading factor that simultaneously regulates CACNA2D3, SFRP2 and CXXC4, and activates the Wnt signalling pathway by interacting with the Gquadruplex motif of EZH2. LncHERES therefore has great potential as a therapy for esophageal cancer as well as other cancers that may be caused by defects in the Wnt signalling pathway [14]. Furthermore, the abundances and stability of IncRNAs that are contained in exosomes lay the foundation for the development of IncRNAs as new tumour biomarkers $[15,16]$. These findings demonstrated that IncRNAs are critically involved in the progression of cancers including esophageal cancer and may serves as biomarkers for diagnosis and prognosis.

The ERK/MAPK pathway is a major driver of oncogenesis and is dysregulated in many human cancers. With the phosphorylate of MAPK1 playing a pivotal role, extracellular signal-regulated kinase $1 / 2$ (ERK1/2) can be activated or upregulated by upstream kinases, transcription factors or differentiation factors. The activated ERK1/2 translocate to the nucleus and then phosphorylate a series of transcription factors such as c-myc, fos, Fra1 and EGR1 [17]. These downstream factors, activated by ERK1/2, further regulate many cell functions, including proliferation, differentiation and transformation [18]. Mechanistically, it is reported that ERK1 and ERK2 can be activated by MEK1 or MEK2 through phosphorylation of the T-E-Y motif in the kinase activation loop [19]. Due to the relative positions of ERK1 and ERK2 in the MAPK pathway, both ERK1 and ERK2 control their output through the activation of their nuclear and cytoplasmic substrates. Moreover, small molecule inhibitors have a clear theoretical basis for this pathway, and high-frequency mutations have been found in many diseases such as colorectal cancer, thyroid cancer and melanoma [20]. However, in terms of current researches, the underlying mechanism between the LncRNA and MAPK1 (ERK2) pathways has not been further elucidation.

In the present study, an unreported IncRNA named GK-IT1 was identified via the analysis of RNA-seq data of the ESCC expression profile of IncRNAs obtained from TCGA. We found that GK-IT1 is highly 
expression in ESCC tissues/cells, and positively correlates with tumour progression, involving clinical staging and patient prognosis. In vivo and in vitro experiments show that GK-IT1 can promote cell growth and metastasis. More importantly, we found that GK-IT1 can regulate the phosphorylation of MAPK1 (ERK2), activating ERK/MAPK1 pathway, thereby contributing to the progression of ESCC. This suggests that GK-IT1 may be an independent prognostic indicator and a promising therapeutic method for ESCC.

\section{Materials And Methods}

\section{Human ESCC tissue specimens}

Fresh paired ESCC tissues used in this study were provided by 80 patients who underwent surgical resection at The First Affiliated Hospital of Chongqing Medical University (Chongqing, China). These tissue samples were immediately stored in liquid nitrogen. All patients signed their informed consent forms and the study was authorised by the Ethics Committee of Chongqing Medical University.

\section{Bioinformatics analysis}

The RNA-seq data for ESCC was downloaded from The Cancer Genome Atlas (TCGA) database, which included 162 cancer tissue samples and 11 adjacent normal tissue samples. The HTSeq-counts data in the database was analysed using $\mathrm{R}$ software (3.6.2). According to the screening conditions of fold change $>2$, P value $<0.05$, and FDR $<0.01,340$ upregulated and 259 downregulated LncRNA were obtained. The survival analysis for TCGA clinical data for ESCC was carried out using SPSS software.

\section{Cell lines and culture conditions}

Five human esophageal squamous cell lines, TE-1, TE-10, ECA-109, Kyse-510 and Kyse-150, in addition to the normal esophageal epithelial cell line, Het-1a, were purchased from the ATCC. Both 293T and 293 cell lines are kept in the laboratory of Chongqing Medical university and were therefore ready to be used.

The TE-10, TE-1,Het-1a,ECA-109, KYSE-510, KESE-150, 293T and 293 cells were all cultured in DMEM medium (Gibco, Carlsad, CA, USA). The cell culture medium contains $10 \%$ fetal bovine serum (FBS, HyClone, Invitgen), $100 \mathrm{U} / \mathrm{ml}$ penicillin and $100 \mathrm{mg} / \mathrm{ml}$ streptomycin and cultured in a humidified incubator containing $5 \% \mathrm{CO}_{2}$ at $37^{\circ} \mathrm{C}$.

\section{Plasmid construction, RNAi and cell transfection}

In order to construct the GK-IT1 overexpression vector, the full length of human GK-IT1 was inserted into the pcDNA3.1-EF1a-mcs-3flag-CMV-GFP vector (Shanghai, China, Hanbio). In order to simulate the sequence without GK-IT1 the vehicle is used as the control. To knock out GK-IT1, siRNA against GK-IT1 (siGK-IT1-1, siGK-IT1-2, siGK-IT1-3) and siRNA-NC against GK-IT1 (Geencam Biotech, Guangzhou, China) were synthesised. The most effective siRNA siGK-IT1-2, detected by the qRT-PCR and GK-IT1 overexpression vectors, was subcloned into a lentiviral vector (hU6-MCS-Ubiquitin-firefly_Luciferase-IRESpuromycin) in order to construct a sh-GK-IT1 carrier, and sh-NC was used as a negative control 
(Guangzhou, Geenchem Biotech). The two helper vectors as well as a lentiviral vector carrying either GKIT1 or sh-NC were transiently transfected into HEK293T cells. After 48h, the virus supernatant was collected, clarified and concentrated for animal research. Puromycin was then used to select stable transfected or infected ESCC cells.

SiRNAs against DUSP6 were purchased from RIBOBIO (Guangzhou, China). The above vectors were then confirmed by sequencing, Si-NC was taken as a control and all transfections were performed with Liposome 2000 (Invitgen, Carlsbad, California), according to the manufacturer's instructions. The sequences of siRNAs and shRNAs in this study are listed in Additional File 1: Table S1.

\section{RNA extraction, nuclear-cytoplasmic fractionation and qRT-PCR assays from tissues or cell lines}

Total RNA was isolated from ESCC cells and paired tissues using TRIzol reagent (Takara, Dalian, China) following the manufacturer's instructions. RNAs from the cytoplasm and nucleus of ESCC cells were separated by the PARIS ${ }^{\text {TM }}$ Kit (Life Technologies, Austin, Texas, USA). Quantitative real-time PCR (qRTPCR) analysis was performed using the TB Green Premix Ex Taq (Takara, Japan). Relative expression levels of genes were quantified by the $2-\Delta \Delta C t$ method. The primer sequences were displayed in Additional File 1: Table S1.

\section{Fluorescence in situ hybridisation (FISH)}

To observe the co-localisation in ESCC cells and paired tissues, FISH assays was performed using the Ribo Fluorescent In Situ Hybridization Kit (RIOBIO, Guangzhou, China) and Cy3-labeled GK-IT1 probe mix was used according to the manufacturer's instructions. Moreover, Cell nuclear were stained with 4,6diamidino-2-phenylindole (DAPI, Beyotime, China). The images were taken under a fluorescence microscope (Leica, Wetzlar, Germany).

\section{Cell proliferation assay}

Colony formation assay, EDU incorporation assay and CCK-8 assay were used to detect the proliferation of esophageal cancer cells. In order to analyse the colony formation, 350 transfected cells / holes were inoculated into 6-well plates and cultured for 2 weeks. The colonies were then fixed with $4 \%$ paraformaldehyde and stained with $0.5 \%$ crystal violet in order to facilitate the counting of the total number of colonies. A commercial kit (RIOBIO, Guangzhou, China) was used for EDU detection (No. C10310) and the manufacturer's instructions were followed, as described earlier. After transfection, the cells from each group were made into cell suspension (density $1 \times 105 / \mathrm{ml}$ ) and inoculated into 96-well plates according to $100 \mathrm{ul} /$ per well. Three multiple holes were set up in each group, and the 96 -well plates were routinely cultured in the incubator. After the cells were stuck to the wall, a 96-well plate was taken out and incubated with CCK-8 solution (Beyotime, shanghai, China) in the incubator for 2 hours. Then the OD value at the absorbance of $450 \mathrm{~nm}$ was measured by an enzyme labelling instrument, and the reading was then recorded. After that, the 96 -well plates were cultured for 24 hours, 48 hours, 72 hours, and 96 hours, respectively, the $\mathrm{OD}$ value was measured, the value recorded and the growth curve drawn using the same method. All tests were carried out in three stages. 


\section{In vitro cell migration and invasion assay}

Horizontal cell migration was examined using wound healing assays. When cell confluency reached the density of approximately $95 \%$, media was ditched and then scratches across the monolayer was perpendicularly scraped by $10 \mu \mathrm{l}$ tip. Cells were washed three times with PBS and then cultured in medium containing 10\% FBS. Using an inverted microscope (Leica, Wetzlar, Germany), the cells were photographed at 0 and $24 \mathrm{~h}$ after wounding. The cell migrative capabilities was represented by the degree of close of the gaps observed under the microscope.

For cell vertical invasion assays, the transfected cells were resuspended in serum-free media at a concentration of $7.5 \times 104 / \mathrm{ml} .500 u$ ul per well complete media was added into 24 -well plates beforehand, then trans well chambers, which were precoated with Corning Matrigel (BD Biocoat, Cat.No. 354234) 6h prior to the experiment, were placed into the well and added with 200ul premixed cell suspension. Further steps were identical, and then $500 \mu$ l of cell culture media supplemented with $10 \%$ FCS was added into the lower chambers and the cells were incubated at $37^{\circ} \mathrm{C}$ in normal cell culture conditions for $16-20 \mathrm{~h}$. Cells that invaded the lower chamber were fixed with methanol for 20 mins at room temperature and stained with $0.1 \%(\mathrm{w} / \mathrm{v})$ crystal violet in the dark. Assays were executed in threefold.

For the cell cycle analysis, cells were harvested and fixed in pre-cold $70 \%$ ethanol at $4^{\circ} \mathrm{C}$ overnight, stained with propidium iodide (PI), and tested with flow cytometry (Becon Dickinson FACS Calibur, NY, USA). Annexin V-FITC and PI staining was then carried out with a flow cytometry to detect the cell apoptosis rate. In addition, TUNEL (Beyotime, Shanghai, China) was used to further detect apoptosis. These images were observed under a fluorescence microscope (Leica, Wetzlar, Germany).

\section{RNA pull-down and mass spectrometry analysis}

Pierce $^{\text {TM }}$ magnetic ribonucleic acid-protein pulldown kit (Thermo Fisher, USA) was used for ribonucleic acid pulldown and mass spectrometry (LC-MS) analysis. In short, we used biotin-labelled antisense RNA and sense RNA (Invitgen) in order to prepare biotin-labelled GK-IT1 by in vitro transcription, according to the manufacturer's instructions. The biotinylated GK-IT1RNA was incubated with streptavidin beads and total cell lysate for $2 \mathrm{~h}$, centrifuged at $4^{\circ} \mathrm{C}$ for 5 mins, and the RNA-protein complex was eluted with a $1 \times$ SDS loading buffer. The protein was separated in a 10\% SDS-PAGE gel and stained with Coomassie blue. The protein bands were cut from the gel and analysed by mass spectrometry.

RNA immunoprecipitation (RIP) assay was performed with a Magna RIP kit (Massachusetts Bilelika Milli Pore), following the manufacturer's guidelines. Briefly, the magnetic beads were incubated with antiMAPK1 antibodies (Abcam, Burlingame, CA, USA) or IgG negative control antibodies (MilliPore, Billerica, MA, USA). Then, cells were lysed and incubated with corresponding antibody-coated beads.

Subsequently, the coprecipitated RNA was extracted using TRIzol regent (Takara, Dalian, China) and the RNA level was detected by qRT-PCR. 
IHC and IF assays were performed as previously reported [21]. For the IF experiment, tissues or cells were incubated with primary antibodies against LC3B (1:200 dilution) (Cell Signalling Technology, Beverly, MA, USA) ,MAPK1(1:200 dilution)(Cell Signalling Technology, Beverly, MA,USA)and P-MAPK1 (1:200 dilution) (Cell Signalling Technology, Beverly, MA, USA) at $4^{\circ} \mathrm{C}$ overnight, then incubated for 1 hours with fluorescein-conjugated secondary antibodies (Alexa Fluor 488) and photographed using a fluorescence microscope (Leica, Wetzlar, Germany). For the IHC assay, paraffin sections were incubated with antibodies against P-MAPK1 (1:200 dilution) and MAPK1(1:200 dilution) (Cell Signalling Technology, Beverly, MA, USA). The images were photographed under an Olympus multifunction microscope (Olympus BX51, Tokyo, Japan)

\section{Western blot analysis}

In short, the proteins were extracted from ESCCs, then quantified by BCA reagents and isolated by $10 \%$ / $12 \%$ SDS-PAGE. After electrophoresis, the protein bands were transferred to PVDF membranes (Bio-Rad, CA, USA). The membranes were blocked with $5 \%$ skim milk and then incubated with primary antibodies against: MAPK1 (1:1000 dilution) (SAB, Santa Cruz Biotechnology, USA); MEK1/2 (1:1000 dilution), PMEK1/2 (1:1000 dilution), P-MAPK1 (1:1000 dilution) (Cell Signaling Technology, Beverly, MA, USA); Cyclin E1 (1:1000 dilution) (Abcam, Burlingame, CA, USA); Bax (1:1000 dilution), Bcl-2 (1:1000 dilution), Caspase-3 cleaved (1:1000 dilution), LC3B (1:1000 dilution), ATG5 (1:1000 dilution), ATG7 (1:1000 dilution), Cyclin D1 (1:1000 dilution), CDK4 (1:1000); and DUSP6 (1:1000 dilution) and GAPDH (1:5000 dilution) (Abcam, Burlingame, CA, USA) overnight at $4^{\circ} \mathrm{C}$, followed by incubating with secondary antibodies (1: 5000 dilution) (Cell Signalling Technology, Beverly, MA, USA) for $1 \mathrm{~h}$. Finally, images of the bands were visualised using chemiluminescence system.

\section{Animal experiments}

For the animal experiments, 4-week-old female BALB/c nude mice were used for xenotransplantation experiments and kept under specific pathogen-free conditions. All operations were approved by the Animal Protection and Utilisation Committee of Chongqing Medical University. TE-10 cells stably transfected with GK-IT1 high expression/mimicking vector or infected with lentivirus carrying sh-GKIT1/sh-NC (Genchem Biotech, Guangzhou) $(2.5 \times 106,200$ ul) were subcutaneously inoculated, and the mice were sacrificed 4 weeks later. The tumour volume was calculated according to the formula (length $\times$ width 2/2), measured once a week, and the tumour weight was determined. Tumour and lung tissue was collected for further research. Lung metastatic were counted under a microscope. On the HEstained tumour section, the micro vessels corresponding to the area with the highest blood vessel density under a microscope were counted. For survival analysis, cells stably transfected with GK-IT1 and mock vector were injected subcutaneously into the mice; the cut-off point for monitoring was two months. 60 days later, the mice that were deemed to still be alive were examined. The mice were then sacrificed and their livers were taken for pathological analysis. In addition, nude mice aged between 8-10 weeks old were bought from the Shanghai Laboratory Animal Company.

\section{Statistical analysis}


Data is presented as the mean \pm S.D. In order to analyse the differences between groups, students' ttest/unpaired two-tailed students' $t$ test, the Mann-Whitney U-test and the $\chi 2$ test were utilised. Survival rates were evaluated using the Kaplan-Meier analysis method and then compared using the Log rank test. HRs and $95 \% \mathrm{Cls}$ were calculated using the Cox proportional hazards model with a p-value $<0.05$ considered statistically significant. Statistical analyses were mainly conducted using SPSS 21.0(IBM, SPSS, Chicago, IL, USA) and GraphPad Prism 6.0 (GraphPad Software Inc., CA, USA).

\section{Results}

\section{GK-IT1 is identified from an online database analysis}

We got a huge number of up-regulated and down-regulated LncRNA by using R software (R3.6.2) to analyse the HTSeq-counts data of esophageal cancer in TCGA database (Fig. 1a-b) and found that LncRNA GK-IT1 was significantly up-regulated and has not been reported (Fig. 1c). Next, we predicted the coding ability of LncRNA GK-IT1 and find that it has the characteristics of long non-coding RNA(Fig. 1d). Then, we analysed the expression of GK-IT1 in gastric cancer (GC), colon cancer (COAD), head and neck squamous cell cancer (HNSC) and esophageal cancer (EC). We found that GK-IT1 is highly expressed in GC, HNSC and EC. Beyond this, we analysed the prognostic significance of GK-IT1 in these cancers. However, GK-IT1 was only predicted poor prognosis in esophageal cancer, while decreased GK-IT1 expression reported better prognosis(Fig. 1E).

\section{GK-IT1 is upregulated in ESCC and associated with poor prognosis}

In China, however, the incidences of esophageal squamous cell carcinoma are much higher than that of esophageal adenocarcinoma, so we focused our attention on esophageal squamous cell carcinoma. Then, RNA-difference analysis of gene expression profiles of 80 ESCC tissues and 11 adjacent nontumour tissues downloaded from TCGA database was identified GK-IT1 as an ESCC-associated IncRNA that may be related to esophageal tumorigenesis. Kaplan- Meier survival curves showed that overall, patients with high expression of GK-IT1 have a poor survival rate and shorter disease free time(Fig. 2a), which suggested that GK-IT1 might play an important role in ESCC. GK-IT1 is derived from X chromosome and it has $397 \mathrm{bp}$ (Fig. 2b). The qRT-PCR analysis was performed to determine the expression of GK-IT1 in 80 pairs of ESCC and its adjacent normal tissues in ESCC patients (Fig. 2C). The results demonstrated that GK-IT1 showed significantly higher expression in tissues than adjacent normal tissues, which was consistent with the results above bioinformatics analysis. what's more, the expression of LncRNA GK-IT1 is positive related to the depth of tumour invasion, lymph node metastasis, TNM stage and tumour size (Fig. 2d-g). A receiver operating characteristic (ROC) curve was further utilised to assess the diagnostic value of GK-IT1 for ESCC screening. According to the ROC curve analysis, at a cut-off value of 7.5 , the area under the curve (AUC) of GK-IT1 was 0.865 , and the specificity and sensitivity value were calculated to be 74.7 and $81.8 \%$. respectively, meaning GK-IT1 was able to sensitively discriminate ESCC from normal tissues (Fig. 2h). ESCC cell lines (TE1, TE10, Eca109, KYSE150 and KYSE510) also exhibited elevated expression in normal esophageal epithelial cells (Het-1a) (Fig. 2i). Subsequently, we evaluated 
the relationship between the expression GK-IT1 and clinical pathological characteristics. The results indicated that the expression of GK-IT1 was positively correlated with T $(P=0.002), N(P=0.02)$ and TNM $(P=0.023) \square$ Table 1区 Furthermore, we also used clinical data of 80 ESCC tissues acquired from TCGA. Correlation of GK-IT1 expression with clinicopathological characteristics in ESCC patients were listed in Table 2. Further analysis was affirmative of the positive association between ESCC expression and T stage $(P=0.032)$ and $N(P=0.04)$ stage. The Cox proportional hazard model was used to evaluate the independent prognostic value of GK-IT1 and it was found that GK-IT1 was a potential independent predictor for a poor prognosis in ESCC patients $(H R=3.329, \mathrm{P}=0.026)$ (Table 3$)$. In short, GK-IT1 could serve as a tumour promoter and high expressions of GK-IT1 might predict a poor prognosis for patients with ESCC. Subsequently, $t$ the subcellular localization of GKIT1 in ESCC cells and tissues was predicted using online website(http://www.csbio.sjtu.edu.cn/bioinf/IncLocator/\#) and subsequently verified by nuclear-cytoplasmic fractionation and FISH assays. (Fig. 2j-k). It was found that GK-IT1 was mainly distributed in the cytoplasm of ESCC cells. 
Table 1

correlation between GK-IT1 expression and clinicopathological features in 70 ESCC patients

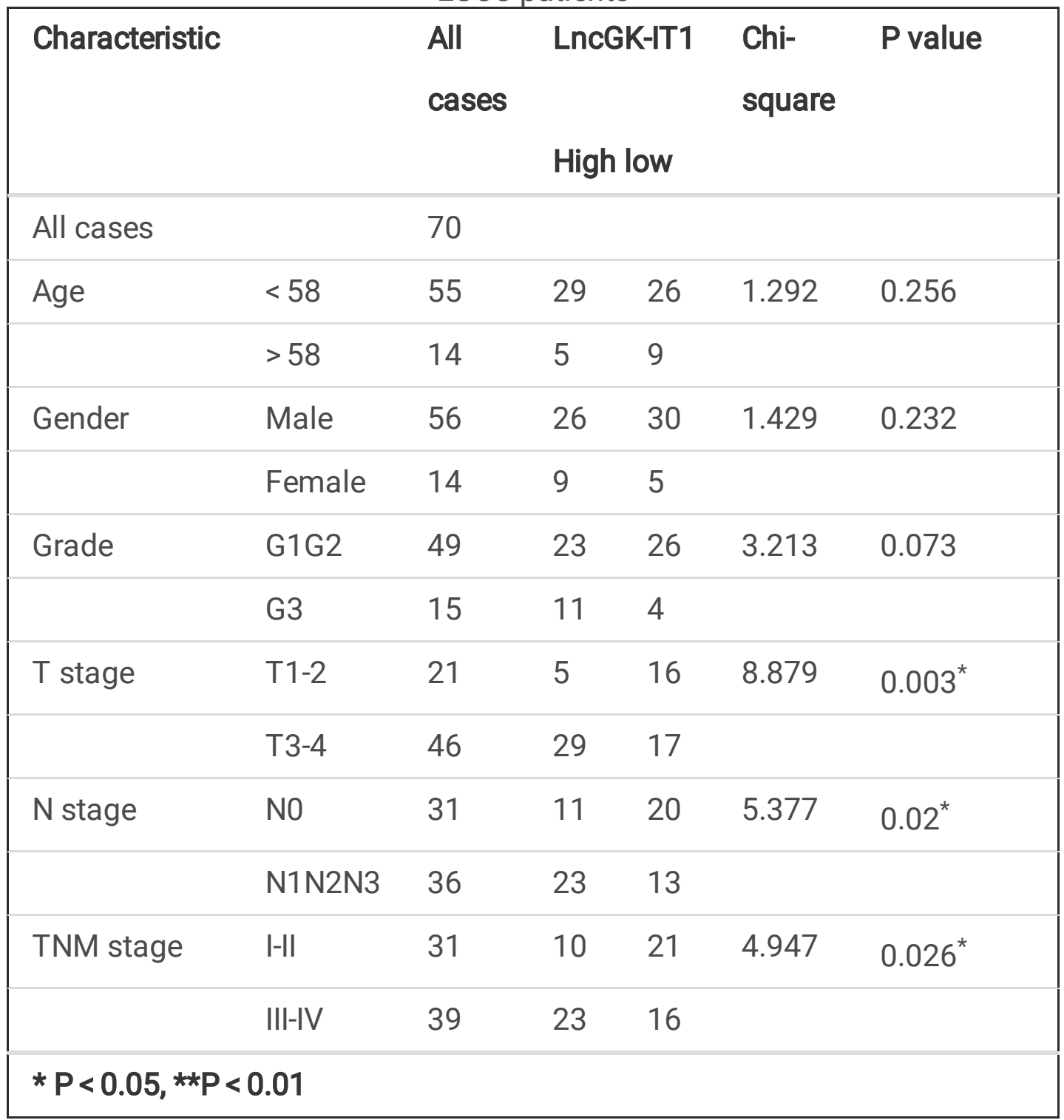


Table 2

correlation between GK-IT1 expression and clinicopathological features in 80 ESCC patients

\begin{tabular}{|c|c|c|c|c|c|c|}
\hline \multirow[t]{2}{*}{ Characteristic } & & & \multicolumn{2}{|c|}{ LncGK-IT1 } & \multirow{2}{*}{$\begin{array}{l}\text { Chi- } \\
\text { square }\end{array}$} & \multirow[t]{2}{*}{$P$ value } \\
\hline & & & \multicolumn{2}{|c|}{ High low } & & \\
\hline All cases & $<58$ & 80 & & & & \\
\hline \multirow[t]{2}{*}{ Age } & $>58$ & 30 & 13 & 17 & 0.853 & 0.356 \\
\hline & Male & 50 & 27 & 23 & & \\
\hline \multirow[t]{2}{*}{ Gender } & Female & 67 & 36 & 31 & 2.296 & 0.13 \\
\hline & $18.5-23.9$ & 13 & 4 & 9 & & \\
\hline \multirow[t]{3}{*}{$\mathrm{BMI}$} & $<18.5$ & 58 & 32 & 26 & 2.257 & 0.133 \\
\hline & $>23.9$ & 4 & 2 & 2 & & \\
\hline & G1G2 & 18 & 6 & 12 & & \\
\hline \multirow[t]{2}{*}{ Grade } & G3 & 51 & 29 & 22 & 1.21 & 0.271 \\
\hline & $\mathrm{T} 1-2$ & 19 & 8 & 11 & & \\
\hline \multirow[t]{2}{*}{ T stage } & T3-4 & 35 & 13 & 22 & 4.575 & $0.032 *$ \\
\hline & NO & 44 & 27 & 17 & & \\
\hline \multirow[t]{2}{*}{ N stage } & N1N2N3 & 43 & 17 & 26 & 4.192 & $0.04^{*}$ \\
\hline & $|-I|$ & 35 & 22 & 13 & & \\
\hline \multirow[t]{2}{*}{ TNM stage } & III-IV & 53 & 24 & 29 & 1.075 & 0.3 \\
\hline & no & 26 & 15 & 11 & & \\
\hline \multirow[t]{2}{*}{ Alcohol use } & yes & 21 & 7 & 14 & 3.464 & 0.063 \\
\hline & no & 56 & 32 & 24 & & \\
\hline Tobacco use & yes & 38 & 16 & 22 & 1.545 & 0.214 \\
\hline
\end{tabular}


Table 3

univariate and multivariate Cox regression analysis of GK-IT1 and survival in patients with ESCC

\begin{tabular}{|llll|}
\hline Clinical variables & \multicolumn{2}{l}{ Univariate analysis } & P value \\
\hline HRE $95 \% \mathrm{Cl}$ & \\
\hline gender & 1.075 & $0.466-2.479$ & 0.865 \\
\hline Tobacco use & 0.227 & $0.051-1.007$ & 0.051 \\
\hline Alcohol use & 2.000 & $0.825-4.850$ & 0.125 \\
\hline Grade (G1 vs G2) & 2.606 & $0.744-9.123$ & 0.134 \\
\hline Grade (G1 vs G3) & 1.356 & $0.687-2.676$ & 0.38 \\
\hline T stage (T1T2 vs T3T4) & 1.343 & $0.593-3.040$ & 0.479 \\
\hline N stage (N0 vs N1N2N3) & 2.642 & $1.071-6.517$ & $0.035^{*}$ \\
\hline TNM(I II vs III IV) & 3.292 & $1.404-7.719$ & $0.006^{*}$ \\
\hline LncRNA GK-IT1 & 3.751 & $1.363-10.320$ & $0.01^{*}$ \\
\hline Clinical variables & Multivariate analysis & P value \\
\hline TNM(I II vs III IV) & 3.166 & $0.780-12.842$ & 0.107 \\
\hline N stage (N0 vs N1N2N3) & 0.848 & $0.188-3.831$ & 0.83 \\
\hline LncRNA GK-IT1 & 3.329 & $1.152-9.623$ & $0.026^{*}$ \\
\hline * P < 0.05, **P<0.01 & & & \\
\hline
\end{tabular}

\section{Altering the expression of GK-IT1 influences proliferation, migration and invasion of ESCC cells}

To probe the potential biological function of GK-IT1 in ESCC, we constructed one overexpression vector for GK-IT1 and designed three siRNAs that targeted gene sequences of GK-IT1. The qRT-PCR results confirmed the overexpression or knockdown efficiency of the indicated vectors or siRNA that is transfected into ESCC cells, respectively. Among the three siRNAs, siGK-IT1-2 and siGK-IT1-3 had the highest silencing efficiency in ESCC cells and thus, in order to avoid off-target effects, two siRNAs with the highest silencing efficiency were selected for further study (Fig.S1a-d). The colony formation, CCK-8 and EdU assays were used for the assessment of cell viability. As shown in results, knockdown of GK-IT1 notably suppressed the viability of 
ESCC cells (Fig. 3a-e), whereas an increase in cell viability was found to be induced due to overexpression of GK-IT1.

Similarly, we found that downregulation of GK-IT1 inhibited the migratory and invasive capabilities of ESCC cells measured by wound- healing and transwell assays (Fig. 4h-i), while upregulation of GK-IT1 revealed an opposite effect. Collectively, these data suggest that GK-IT1 plays a vital role in the oncogenesis and malignant progression of ESCC cells.

\section{Knock down of GK-IT1 regulates cell cycle progression, apoptosis and autophagy in ESCC}

For the purpose of exploring the role of GK-IT1 in ESCC progression, after GK-IT1 knockdown in vitro, the cell cycle, apoptosis and autophagy were all investigated. The cell cycle analysis showed that the silencing of GK-IT1 increased ESCC cells distributions in the G1 phase while cells in the S phase were remarkably reduced, which suggested that arrestment of cell cycle at the $\mathrm{G} 1$ phase was induced by si-GKIT1 (Fig. 4a).Moreover, western blot assay demonstrated that the downregulation of GK-IT1 decreased the abundances of cell cycle-related proteins, which leads to the block of cell cycle progression in ESCC cells (Fig. 4d).

Then, the implications of GK-IT1 knockdown on apoptosis of ESCC cells was assessed. TUNEL assay indicated that the downregulation of GK-IT1 markedly increased the percentage of the TUNEL positive cells in comparison with control groups (Fig. 4b). Furthermore, Flow cytometry analysis through Annexin V/PI double staining exhibited that the apoptotic rates of ESCC cells in the si-GK-IT1 group were significantly elevated compared to those of the si-NC cells (Fig. 4c). In addition, the expressions of apoptosis-related proteins were determined by western blotting. Consistent with the above results, the significantly enhanced expressions of activated (cleaved) caspase-3 and proapoptotic protein Bax and decreased expression of $\mathrm{Bcl}-2$ were observed in ESCC cells transfected with si-GK-IT1, while overexpression of GK-IT1 showed the opposite results (Fig. 4e). Subsequently, we examined the effect of GK-IT1 knockdown on the autophagic status of ESCC cells. Immunofluorescence assay showed that depletion of GK-IT1 activated cellular autophagy, as evidenced by inducement of LC3- II punctuation and an accumulation of autophagosomes. There were significantly more LC3-II puncta per cell in the si-GK-IT1 group than in the si-NC ESCC cells (Fig. 5f). The conversion from LC3-I to LC3-II markedly increased and the levels of autophagy-related proteins ATG5 and ATG7 were significantly enhanced by the knockdown of GK-IT1, as shown by the western blot results (Fig. $5 \mathrm{~g}$ ). Taken together, these results suggest that GKIT1 might regulate the cell cycle, apoptosis and autophagy in ESCC cells.

\section{Gk-it1 Interacts With Mapk1}

Since the proportions of GK-IT1 was predominantly located in the cytoplasm (Fig. 2I-k), we next focused on clarifying its potential functions with respect to cytoplasmic location in ESCC cells. Considering the ability of IncRNAs to bind macro-biomolecules, we investigated if it's the case for our specific IncRNAs. As dually evidenced by both RNA pull-down assay and mass spectrometry (MS) analysis, GK-IT1 was proved to be able to bind 64 proteins (Fig. 5a-c). Among the identified proteins, several have been reported to have RNA binding capacity, including PABPC1, PEBP1, as well as proteins in the cell cycle kinase family, 
such as CDK1, which thus supports the validity of our approach. One particular candidate protein Mitogen-activated protein kinase 1(MAPK1) aroused our interests because of the important role of ERK/MAPK signalling in the cancer progression. Similarly, the binding ability of LncRNA GK-IT1 and MAPK1 was predicted by RPIseq online website(http://pridb.gdcb.iastate.edu/RPISeq/), and the two algorithms of RF, SVM are used to analysis, the results were both positive (Fig. $5 \mathrm{~d}$ ). The results of RNA pull-down assay and western blotting experiments confirmed the interactions between GK-IT1 and MAPK1. Western blotting experiment showed that the content of MAPK1 protein in the experimental group was higher than that in the control (Fig. 5e). As an important complement to above experiments, RNA Binding Protein Immunoprecipitation (RIP) assay was performed to further validate the binding of MAPK1 to GK-IT1 as an independent approach. the qRT-PCR following RNA immunoprecipitation assays and Agarose gel electrophoresis confirmed an enrichment of GK-IT1 in the complex containing ERK2, compared with IgG control (Fig. 5f-g). In addition, we used FISH and IF experiments to verify the subcellular co-localization of GK-IT1 and MAPK1. We found that GK-IT1 and MAPK1 were mainly distributed in the cytoplasm. (Fig. 5h).

\section{GK-IT1 promotes ESCC progression via activation of ERK/MAPK signalling pathways}

Next, we explored the contribution of GK-IT1 to the regulation of the ERK/MAPK signalling pathways. Notably, GK-IT1 depletion significantly attenuated the phosphorylation of ERK1/2, while overexpression of GK-IT1 led to increased phosphorylated ERK1/2 level. In contrast, MAPK1 levels and phosphorylation levels of MEK1/2 were unchanged upon the silencing of GK-IT1 (Fig. 6a). MAPK1 is phosphorylated by MEK1/2 whereas its dephosphorylation is mediated by phosphatases, such as Dual Specificity Phosphatase 6 (DUSP-6) in the cytoplasm. We postulated that the interaction between GK-IT1 and MAPK1 could regulate the phosphorylation of MAPK1, since the phosphorylation of MEK $1 / 2$ was unaffected by neither the overexpression nor the knockdown of GK-IT1.

To address this hypothesis, we sought to explore if DUSP-6 was responsible for GK-IT1-mediated dephosphorylation of MAPK1 in ESCC cell lines. Specifically, RNAi-mediated knockdown of DUSP-6 prominently elevated the level of phosphorylated MAPK1 (Fig. 6b). Then, the western blot analysis exhibited that when si-GK-IT1 and si-DUSP-6 were co-transfected, rather than only si-GK-IT1, the phosphorylation level in MAPK1 moderately increased (Fig. 6c).

As a MAPK phosphatase, DUSP- 6 exerts critical effect on the negative regulation of MAPK1 phosphorylation. A recent study reported that DUSP- 6 was downregulated and to suppress tumour progression in ESCC. Furthermore, it was reported that DUSP-6 had a certain suppressive effect on ESCC progression via inactivating the ERK/MAPK signalling pathway. To further explore that GK-IT1 affects the functions of esophageal squamous cell carcinoma cells by regulating MAPK1 phosphorylation, we respectively transfected si-NC, si-GK-IT1 and si-GK-IT1 + si-DUSP-6. We found that knocking down GK-IT1 inhibited the proliferation, invasion and migration of esophageal squamous cell carcinoma cells. But knocking down GK-IT1 and DUSP-6 at the same time can reverse this trend (Fig. 6d-i). this demonstrated 
both GK-IT1 and Dusp-6 regulate cell function by affecting the phosphorylation of MAPK1. collectively, we found that GK-IT1 bound directly to MAPK1, to mediate the phosphorylation of MAPK1.

\section{Gk-it1 Can Promote Xenograft Tumour Growth And Metastasis}

In order to investigate the effect of LNC GK-IT1 on tumour growth and metastasis in vivo, a xenografted tumour model of human esophageal squamous cell carcinoma was established. TE-10 cells stable overexpressing or silencing GK-IT1 and corresponding control cells were subcutaneously injected into female nude mice to observe the effect of GK-IT1 on tumour growth and metastasis in nude mice, and to explore the effect of GK-IT1 on the growth and metastasis of human esophageal squamous cell carcinoma. The results showed that the tumour volume and weight of GK-IT1 overexpression group were significantly higher than those of control group, while the tumour growth of GK-IT1 knockout group was significantly inhibited (Fig. 7a-c).

Subsequently, we further investigated the effect of GK-IT1 on the expression of ERK/MAPK1 pathway related proteins in vivo. Western blotting results showed that the expression of P-MAPK1 was significantly increased or decreased in GK-IT1 overexpressed or silenced xenograft tumour tissues compared with the control group (Fig. 7d-e), IHC analysis further showed that overexpression of GK-IT1 is associated with elevated expression of P-MAPK1, while silence GK-IT1 had the opposite effect(Fig,7f).In the survival experiment, the Kaplan-Meier survival curve showed that the overall survival rate of nude mice injected with GK-IT1 overexpressed TE-10 cells was lower than that in the control group, and the difference was statistically significant (Fig. $7 \mathrm{~g}$ ). the number and size of liver and lung metastatic nodules in the GK-IT1 overexpression group were higher than those in the control group (Fig. 7h-i). In addition, these results are consistent with the results of vitro experiments, suggesting that GK-IT1 may promote the occurrence and metastasis of esophageal squamous cell carcinoma by activating ERK/MAPK pathway.

\section{Discussion}

Esophageal cancer affects more than 450,000 people and is still the sixth leading cause of cancer-related deaths in the world. However, neither chemotherapy nor radiotherapy are effective in treating esophageal cancer, especially esophageal squamous cell carcinoma [22]. Therefore, developing new therapeutic methods to improve the prognosis of ESCC patients is urgency. In recent years, the involvement of IncRNAs in tumorigenesis and tumour progression has gained widespread attention. the purpose of this study is to explore the carcinogenic mechanism of LnCRNA GK-IT1 on EC, and especially on ESCC.

In this study, we take an analysis the LncRNA expression profile of the ESCC from the TCGA database. Then we concentrated on exploring the potential role and mechanism of a new LncRNA termed GK-IT1 which was found to be upregulated in ESCC and was notably associated with the clinical stage and poor prognosis of ESCC patients. Beside this, we found that the knockdown of GK-IT1 in ESCC could significantly lead to the suppression of cell proliferation and invasion, induce cell apoptosis and autophagy as well as restrain oncogenesis and metastasis in vivo while overexpressing GK-IT1 caused the opposite effects. Mechanistically, we indicated that GK-IT1 might mediate the phosphorylation of 
MAPK1, which activates the MEK/MAPK signalling cascade and promotes the tumorigenesis of ESCC. Our data elucidated that GK-IT1 played an oncogenic role in the progression of ESCC and potentially might be developed as a new diagnostic and prognostic marker for ESCC patients.

LncRNA works through a number of different mechanisms. Many studies are focused on the "ceRNA" mechanism. For example, Wang et al. found that IncTUG1 enhances the radio resistance of ESCC by lowering the level of miR-1443p and regulating the MET/EGFR/AKT axis [23]. Meanwhile, Hao et al. clarified the carcinogenic role of the CCDC144NL-AS1/miR-143-3p/MAP3K7 axis in the progression of gastric cancer [24]. additionally, lots of IncRNAs can also regulate RNA-binding proteins to influence cancer progression, including RMST [25], HCG22 [26] and SNHG14 [27]. Here, we identified and verified several interacting protein partners of GK-IT1, including CDK1, MAPK1 and PABPC1. Notably, PABPC1 has been observed to possess RNA-binding activity [28]. Since PABPC1 also increases the stability of mRNAs or LncRNAs, the association between GK-IT1 and PABPC1 suggests the likelihood that this IncRNA might be linked to multiple cellular processes [29]. In addition, our results showed that a small proportion of GKIT1 was distributed in the nucleus, and that cyclin-dependent kinases such as CDK1 were identified to be potentially interacting with GK-IT1. Collectively, those data thus imply that GK-IT1 may have additional roles in the range of nucleus. However, it has not been fully studied that the IncRNAs are involved in the regulation of signalling pathways.

ERK/MAPK pathway is one of the key signalling pathways that facilitate proliferation and survival of cancer cells. Furthermore, they are also involved in biological processes such as apoptosis [30], autophagy [31], EMT [32] and even the Warburg Effect [33]. For instance, in a hypoxic microenvironment, a noticeable link has been found between YAP transcriptional activation and hypoxic glycolysis governed by the ERK2-dependent 14-3-3 3 Ser37 [34]. The phosphorylation for malignant progression in MEK1/2 activates human ERK1/2 by first catalysing the phosphorylation of Y204/187 and then T202/185, both residues of which occur within the activation segment. The phosphorylation of both residues is required for enzyme activation. The only Raf substrates are MEK $1 / 2$ and the only MEK $1 / 2$ substrates are ERK $1 / 2$. In contrast, ERK1/2 can catalyse the phosphorylation of many cytoplasmic and nuclear substrates including transcription factors and regulatory molecules [35]. However, it is not completely understood the mechanism of action regarding the regulation of these pathways by RNA molecules. In this research, we demonstrated that GK-IT1 regulated the ERK/MAPK pathways by mediating the phosphorylation of MAPK1. Then, the phosphorylated MAPK1 enters the nucleus and promotes the malignant progression of the tumour in a variety of ways, as mentioned earlier.

DUSP-6 is an important phosphatase for the mitogen-activated protein (MAP) kinase superfamily (MAPK/ERK, SAPK/JNK, p38), which negatively regulates protein (MAP) kinase phosphorylation through inactivating their target kinases by dephosphorylating both the phosphoserine/threonine and phosphor tyrosine residues. DUSP-6 was found to be downregulated and inhibit tumour development in ESCC [36]. Recent studies reported that lincRNA PICSAR can regulate the expression of DUSP-6 to affect the MAPK/ERK pathway and promote the growth of skin squamous cell carcinoma [37], which suggests that DUSP-6 may play a role in cancers by regulating the dephosphorylation of MAPK1. To further clarify the 
underlying mechanism between GK-IT1 and phosphorylated MAPK1, we knocked out DUSP-6, thus promoting the phosphorylation of MAPK1. Then we also knocked out GK-IT1 with Knock out DUSP-6 or not, thus further confirming whether GK-IT1 exerts its cancer-promoting biological function by regulating the phosphorylation of MAPK1.The results clarified that GK-IT1 can rescue the effect of DUSP-6 on dephosphorylating MAPK1. But whether GK-IT1 has the same site with DSUP-6 so that competitive with each other on modulating the phosphorylation of MAPK1 or they have the completely distinct Mechanism each other for the phosphorylation of MAPK1, we remain furthermore study.

\section{Conclusion}

In conclusion, we found an unreported LncRNA, termed GK-IT1, which could play an oncogenic role in ESCC and is associated with a poor prognosis. We also found that GK-IT1 might stimulate the phosphorylation of MAPK1 and then mediate the ERK/MAPK signalling pathway, leading to oncogenesis and progression of ESCC. our data does suggest that GK-IT1 can act as a promising prognostic biomarker and new therapeutic target for ESCC. However, the specific targets for the binding of GK-IT1 and MAPK1 and how to affect their phosphorylation activity still need further study.

\section{Abbreviations}

LncRNA: Long non-coding RNA; GK-IT1: GK intronic transcript 1;ESCC: esophageal squamous cell carcinoma; MAPK1(ERK2) :mitogen activated protein kinase 1;MEK: MAP kinase-ERK kinase;DUSP-6:dual specificity phosphatase 6;RNA-seq: RNA sequencing; qRT-PCR: Real-time quantitative polymerase chain reaction ; TCGA: The Cancer Genome Atlas; FISH: Fluorescence in situ hybridization; IHC: Immunohistochemistry; IF: Immunofluorescence; 3'UTR: 3'-untranslated region; RIP: RNA immunoprecipitation; ROC curve: Receiver operating characteristic curve; RNAi: RNA interfere

\section{Declarations}

\section{Acknowledgements}

We thank Department of thoracic surgery, The First Affiliated Hospital of Chongqing Medical University, Chongqing, China for providing the ESCC tissue samples and related anonymous clinical data.

\section{Funding}

This work was supported by Chongqing Medical University of China (NO. XBYB2007022) and the project of Research on the Construction of helicopter Emergency Medical Rescue training system of The First Affiliated Hospital of Chongqing Medical University (NO.2018MSXM149).

\section{Availability of data and materials}


The datasets used and analysed during the current study are available from the corresponding author on reasonable request.

\section{Ethics approval and consent to participate}

The study was authorized by the Ethics Committee of The First Affiliated Hospital of Chongqing Medical University. All patients signed consent forms. The animal protocols were approved by Chongqing Medical University Animal Care and Use Committee (approval ID: 2020-732).

\section{Competing interests}

The authors declare that they have no competing interests.

\section{Consent for publication}

All authors give consent for the publication of the manuscript in Journal and Experimental and Clinical Cancer Research

\section{Authors' contributions}

LC and LX designed the research and supervised the project. XY executed all experiments. $X Y, T Y Z$ and HWL performed statistical analysis of data. ZYL and TT were responsible for clinical sample collection and HMT provided support experimental technical support. LC and XY wrote the manuscript. All authors read and approved the final manuscript.

\section{Author details}

${ }^{1}$ Department of thoracic surgery, The First Affiliated Hospital of Chongqing Medical University, Chongqing, China. University, \#1 Youyi Rd, Chongqing 400016, China. ${ }^{2}$ the Frist People's Hospital of Chongqing Liang jiang New Area, Chongqing 410020, China. ${ }^{3}$ Department of Cell Biology and Genetics, Chongqing Medical University, \#1Yixueyuan Road, Chongqing 400016, China . ${ }^{4}$ Department of Endocrine and breast surgery, The First Affiliated Hospital of Chongqing Medical University, \#1 Youyi Rd, Chongqing 400016, China.

\section{References}

[1] BRAY F, FERLAY J, SOERJOMATARAM I, et al. Global cancer statistics 2018: GLOBOCAN estimates of incidence and mortality worldwide for 36 cancers in 185 countries [J]. CA Cancer J Clin, 2018, 68(6): 394-424.

[2] JEMAL A, BRAY F, CENTER M M, et al. Global cancer statistics [J]. CA Cancer J Clin, 2011, 61(2): 69-90. 
[3] ARNOLD M, SOERJOMATARAM I, FERLAY J, et al. Global incidence of oesophageal cancer by histological subtype in 2012 [J]. Gut, 2015, 64(3): 381-7.

[4] ARNOLD M, LAVERSANNE M, BROWN L M, et al. Predicting the Future Burden of Esophageal Cancer by Histological Subtype: International Trends in Incidence up to 2030 [J]. Am J Gastroenterol, 2017, 112(8): 1247-55.

[5] JOHNSSON P, LIPOVICH L, GRANDéR D, et al. Evolutionary conservation of long non-coding RNAs; sequence, structure, function [J]. Biochimica et Biophysica Acta (BBA) - General Subjects, 2014, 1840(3): 1063-71.

[6] LI W, ZHENG J, DENG J, et al. Increased levels of the long intergenic non-protein coding RNA POU3F3 promote DNA methylation in esophageal squamous cell carcinoma cells [J]. Gastroenterology,2014, 146(7):1714-26.e5.

[7] KARRETH FLORIAN A, TAY Y, PERNA D, et al. In Vivo Identification of Tumor- Suppressive PTEN ceRNAs in an Oncogenic BRAF-Induced Mouse Model of Melanoma [J]. Cell, 2011, 147(2): 382-95.

[8] PRENSNER J R, IYER M K, SAHU A, et al. The long noncoding RNA SChLAP1 promotes aggressive prostate cancer and antagonizes the SWI/SNF complex [J]. Nat Genet, 2013, 45(11): 1392-8.

[9] HUARTE M, GUTTMAN M, FELDSER D, et al. A Large Intergenic Noncoding RNA Induced by p53 Mediates Global Gene Repression in the p53 Response [J]. Cell, 2010, 142(3): 409-19.

[10] GUPTA R A, SHAH N, WANG K C, et al. Long non-coding RNA HOTAIR reprograms chromatin state to promote cancer metastasis [J]. Nature, 2010, 464(7291): 1071-6.

[11] HUNG C L, WANG L Y, YU Y L, et al. A long noncoding RNA connects c-Myc to tumor metabolism [J]. Proc Natl Acad Sci U S A, 2014, 111(52): 18697-702.

[12] SHAN T D, TIAN Z B, LI Q, et al. Long intergenic noncoding RNA 00908 promotes proliferation and inhibits apoptosis of colorectal cancer cells by regulating KLF5 expression [J]. J Cell Physiol,2021, 236(2):889-899.

[13] HAN M, QIAN X, CAO H, et al. IncRNA ZNF649-AS1 Induces Trastuzumab Resistance by Promoting ATG5 Expression and Autophagy [J]. Molecular Therapy, 2020, 28(11): 2488-502.

[14] YOU B H, YOON J H, KANG H, et al. HERES, a IncRNA that regulates canonical and noncanonical Wnt signaling pathways via interaction with EZH2 [J]. Proc Natl Acad Sci U S A, 2019, 116(49): 24620-9.

[15] QU L, DING J, CHEN C, et al. Exosome-Transmitted IncARSR Promotes Sunitinib Resistance in Renal Cancer by Acting as a Competing Endogenous RNA [J]. Cancer Cell, 2016, 29(5): 653-68. 
[16] GEZER U, OZGUR E, CETINKAYA M, et al. Long non-coding RNAs with low expression levels in cells are enriched in secreted exosomes [J]. Cell Biol Int, 2014, 38(9): 1076-9.

[17] COOK S J, STUART K, GILLEY R, et al. Control of cell death and mitochondrial fission by ERK1/2 MAP kinase signalling [J]. FEBS J, 2017, 284(24): 4177-95.

[18] LAKE D, CORRêA S A L, MüLLER J. Negative feedback regulation of the ERK1/2 MAPK pathway [J]. Cellular and Molecular Life Sciences, 2016, 73(23): 4397-413.

[19] DHILLON A S, HAGAN S, RATH O, et al. MAP kinase signalling pathways in cancer [J]. Oncogene, 2007, 26(22): 3279-90.

[20] HANAHAN D, WEINBERG R A. Hallmarks of cancer: the next generation [J]. Cell, 2011, 144(5): 64674.

[21] DONG F, RUAN S, WANG J, et al. M2 macrophage-induced IncRNA PCAT6 facilitates tumorigenesis and angiogenesis of triple-negative breast cancer through modulation of VEGFR2 [J]. Cell Death Dis, 2020, 11(9): 728.

[22] CHUANG-XIN L, WEN-YU W, YAO C, et al. Quercetin enhances the effects of 5-fluorouracil-mediated growth inhibition and apoptosis of esophageal cancer cells by inhibiting NF-kappaB [J]. Oncol Lett, 2012, 4(4): 775-8.

[23] WANG P, YANG Z, YE T, et al. IncTUG1/miR-144-3p affect the radiosensitivity of esophageal squamous cell carcinoma by competitively regulating c-MET [J]. J Exp Clin Cancer Res, 2020, 39(1): 7.

[24] FAN H, GE Y, MA X, et al. Long non-coding RNA CCDC144NL-AS1 sponges miR-143-3p and regulates MAP3K7 by acting as a competing endogenous RNA in gastric cancer [J]. Cell Death \& Disease, 2020, 11(7):

[25] PENG W X, KOIRALA P, ZHANG W, et al. IncRNA RMST Enhances DNMT3 Expression through Interaction with HuR [J]. Mol Ther, 2020, 28(1): 9-18.

[26] JIANG D, ZHANG Y, YANG L, et al. Long noncoding RNA HCG22 suppresses proliferation and metastasis of bladder cancer cells by regulation of PTBP1 [J]. J Cell Physiol, 2020, 235(2): 1711-22.

[27] ZHANG H, XU H B, KURBAN E, et al. LncRNA SNHG14 promotes hepatocellular carcinoma progression via H3K27 acetylation activated PABPC1 by PTEN signaling [J]. Cell Death Dis, 2020, 11(8): 646.

[28] Su R, Ma J, Zheng J, et al. PABPC1-induced stabilization of BDNF-AS inhibits malignant progression of glioblastoma cells through STAU1-mediated decay [J]. Cell Death Dis, 2020, 11(2):81. 
[29] SU R, MA J, ZHENG J, et al. PABPC1-induced stabilization of BDNF-AS inhibits malignant progression of glioblastoma cells through STAU1-mediated decay [J]. Cell Death Dis, 2020, 11(2): 81.

[30] RAHMAN A, PALLICHANKANDY S, THAYYULLATHIL F, et al. Critical role of $\mathrm{H} 2 \mathrm{O} 2$ in mediating sanguinarine-induced apoptosis in prostate cancer cells via facilitating ceramide generation, ERK1/2 phosphorylation, and Par-4 cleavage [J]. Free Radic Biol Med,2019, 134:527-544.

[31] LEONARDI M, PERNA E, TRONNOLONE S, et al. Activated kinase screening identifies the IKBKE oncogene as a positive regulator of autophagy [J]. Autophagy, 2019, 15(2): 312-26.

[32] SHIN S, BUEL G R, NAGIEC M J, et al. ERK2 regulates epithelial-to-mesenchymal plasticity through DOCK10-dependent Rac1/FoxO1 activation [J]. Proc Natl Acad Sci U S A, 2019, 116(8): 2967-76.

[33] YANG W, ZHENG Y, XIA Y, et al. ERK1/2-dependent phosphorylation and nuclear translocation of PKM2 promotes the Warburg effect [J]. Nat Cell Biol, 2012, 14(12): 1295-304.

[34] JIA Y, LI H Y, WANG J, et al. Phosphorylation of 14-3-3zeta links YAP transcriptional activation to hypoxic glycolysis for tumorigenesis [J]. Oncogenesis, 2019, 8(5): 31.

[35] MISSINATO M A, SAYDMOHAMMED M, ZUPPO D A, et al. Dusp6 attenuates Ras/MAPK signaling to limit zebrafish heart regeneration [J]. Development, 2018, 145(5):

[36] MA J, YU X, GUO L, et al. DUSP6, a tumor suppressor, is involved in differentiation and apoptosis in esophageal squamous cell carcinoma [J]. Oncol Lett, 2013, 6(6): 1624-30.

[37] Piipponen M, Nissinen L, Farshchian M, et al. Long Noncoding RNA PICSAR Promotes Growth of Cutaneous Squamous Cell Carcinoma by Regulating ERK1/2 Activity [J]. J Invest Dermatol. 2016, 136(8):1701-1710.

\section{Figures}


A.

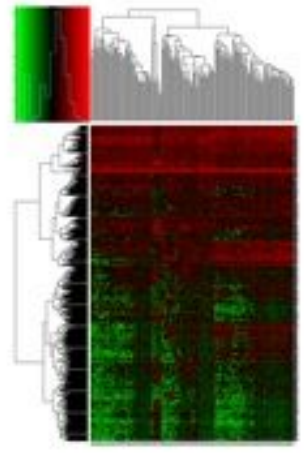

B.

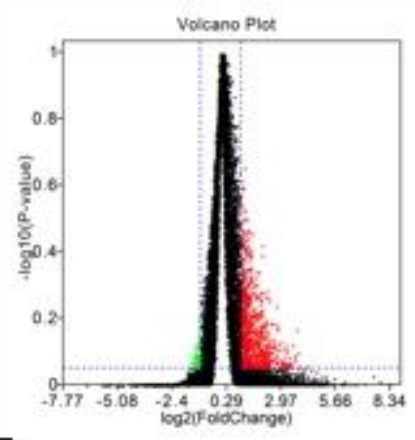

C.

\begin{tabular}{ll}
\hline Name & Fold Change \\
\hline LINC00392 & 3.339 \\
LUCAT1 & 2.891
\end{tabular}

GK-IT1 2.311

CASC9 2.388

LINC00152 2.236

\begin{tabular}{|l|l|}
\hline Sequence name & ENST00000441146.1 \\
\hline RNA size & 397 \\
\hline ORF size & 78 \\
\hline Coding probability & 0.01323 \\
\hline Coding label & No \\
\hline
\end{tabular}
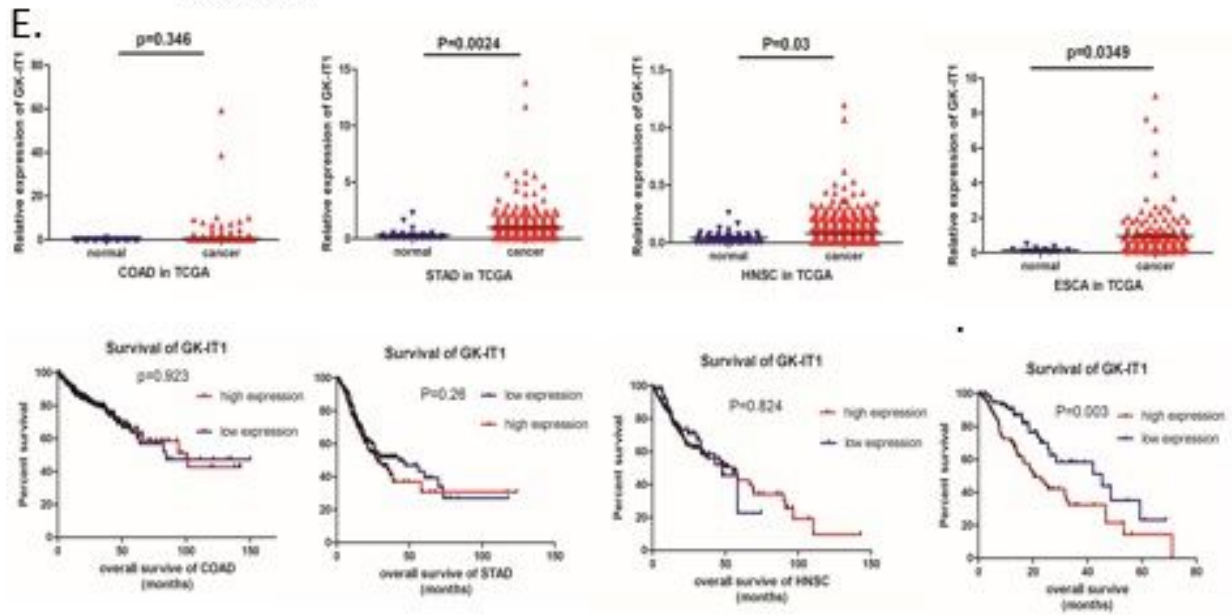

Figure 1

GK-IT1 is upregulated in EC tissues and associated with poor prognosis a and b the heat map and scatter map were obtained through the difference analysis of the data of esophageal cancer in TCGA. c Some LncRNAs were significantly up-regulated in esophageal cancer in esophageal cancer. d CPAD analysis of GK-IT1. e GK-IT1 expression was detected in COAD, STAD, HNSC and ESCA and Kaplan-Meier survival analysis of the overall survival in two groups defined by low and high expression of GK-IT1 in patients with COAD, STAD, HNSC and ESCC. The median expression of GK-IT1 was used as cut-off. $p=0.923 \square$ $p=0.26, p=0.824$ and $p=0.003$ by Long-rank test. 
A
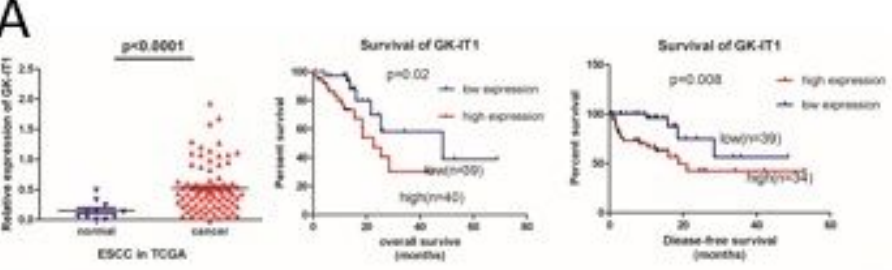

B
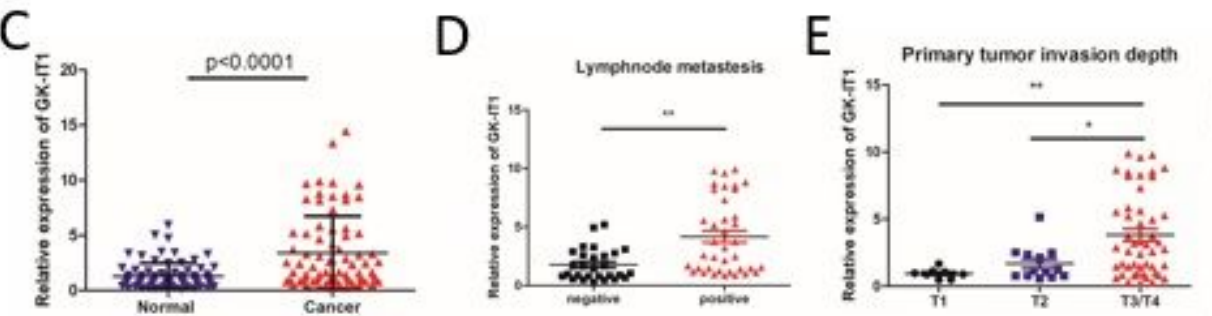

$\mathrm{F}$

G
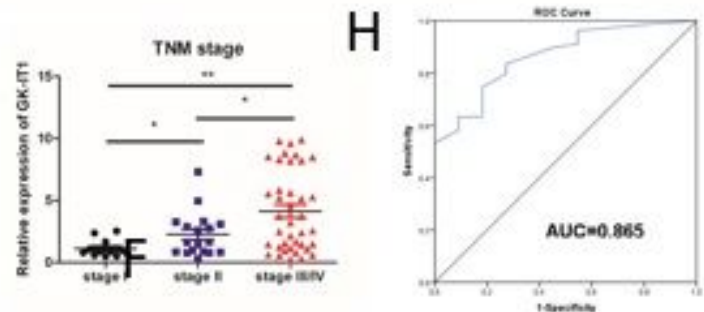

$\mathrm{M}$
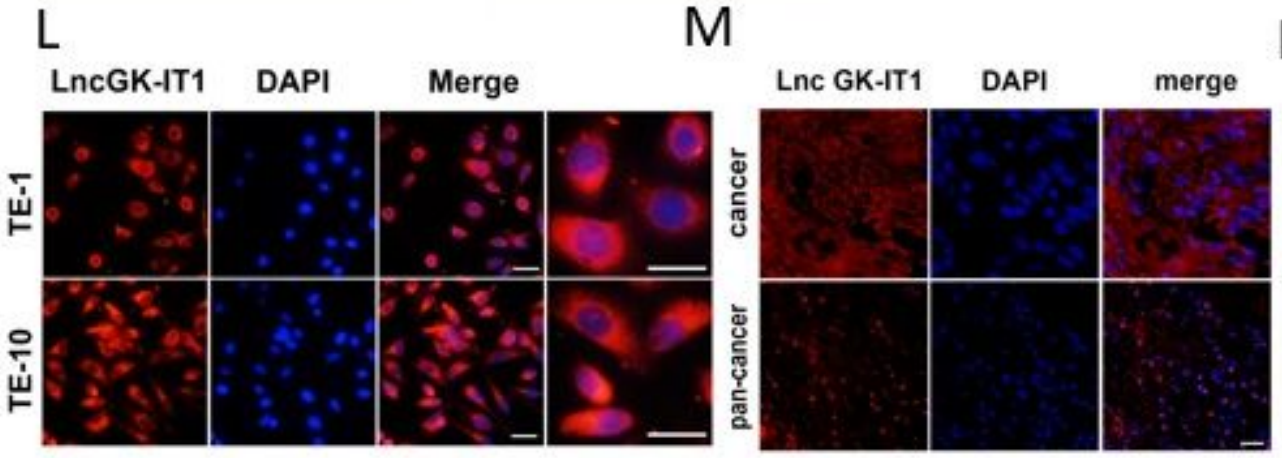

$\mathrm{K}$

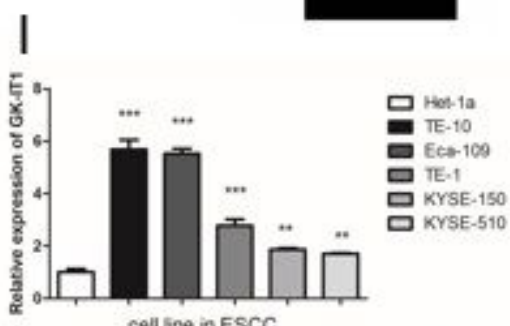

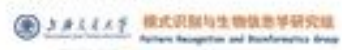
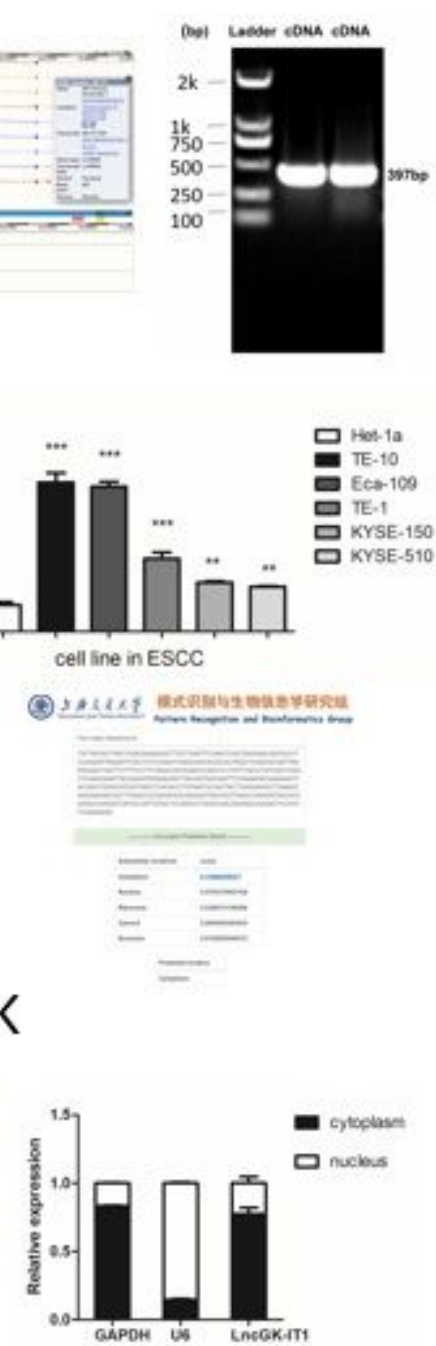

Figure 2

GK-IT1 is upregulated in ESCC tissues and associated with clinical progression a LncRNA GK-IT1 expression was detected in ESCC. Kaplan-Meier survival analysis of the Overall Survival and DiseaseFree Survival in two groups defined by low and high expression of GK-IT1 in patients with ESCC. The median expression of GK-IT1 was used as cut-off. $p=0.02$ and $p=0.008$ by Log-rank test. $b$ LncRNA GKIT1 derived from X chromosome (https://asia.ensembl.org/index.html). the sequences and RT-PCR of GKIT1 was detected by agarose gel electrophoresis. c Comparison of GK-IT1 in ESCC patient's tissues ( $\mathrm{n}=$ 78) and normal tissues ( $\mathrm{n}=78$ ) by RT-qPCR. $\mathrm{d}$-g. The expression of GK-IT1 was determined by RT-PCR at different primary tumour invasion depth, lymph node metastasis, TNM stages, Tumour size, of ESCC patients compare with corresponding adjacent normal tissues. h. ROC curve was applied to evaluate the diagnostic value of GK-IT1 for ESCC patients. i Relative expression of GK-IT1 in five ESCC cell lines (TE10, TE-1, Eca-109, KYSE-150, KYSE-510) and an esophageal epithelial cell(Het-1a). j The subcellular localization of GK-IT1 was predicted by online website(http://www.csbio.sjtu.edu.cn/bioinf/IncLocator/\#). k Nuclear-cytoplasmic fractionation assay 
indicated that LncRNA GK-IT1 was mainly localized in the cytoplasm of ESCC cells. I-m. The localization of GK-IT1was observed in ESCC tissues (magnification, $\times 100$, Scale bar,50 $\mu \mathrm{m}$ ) and cells (magnification, $\times 200$, Scale bar, $50 \mu \mathrm{m})$ by FISH. The nuclear were stained with DAPI. The data are presented as the mean $\pm S D, * * P<0.01, * \star * P<0.001$

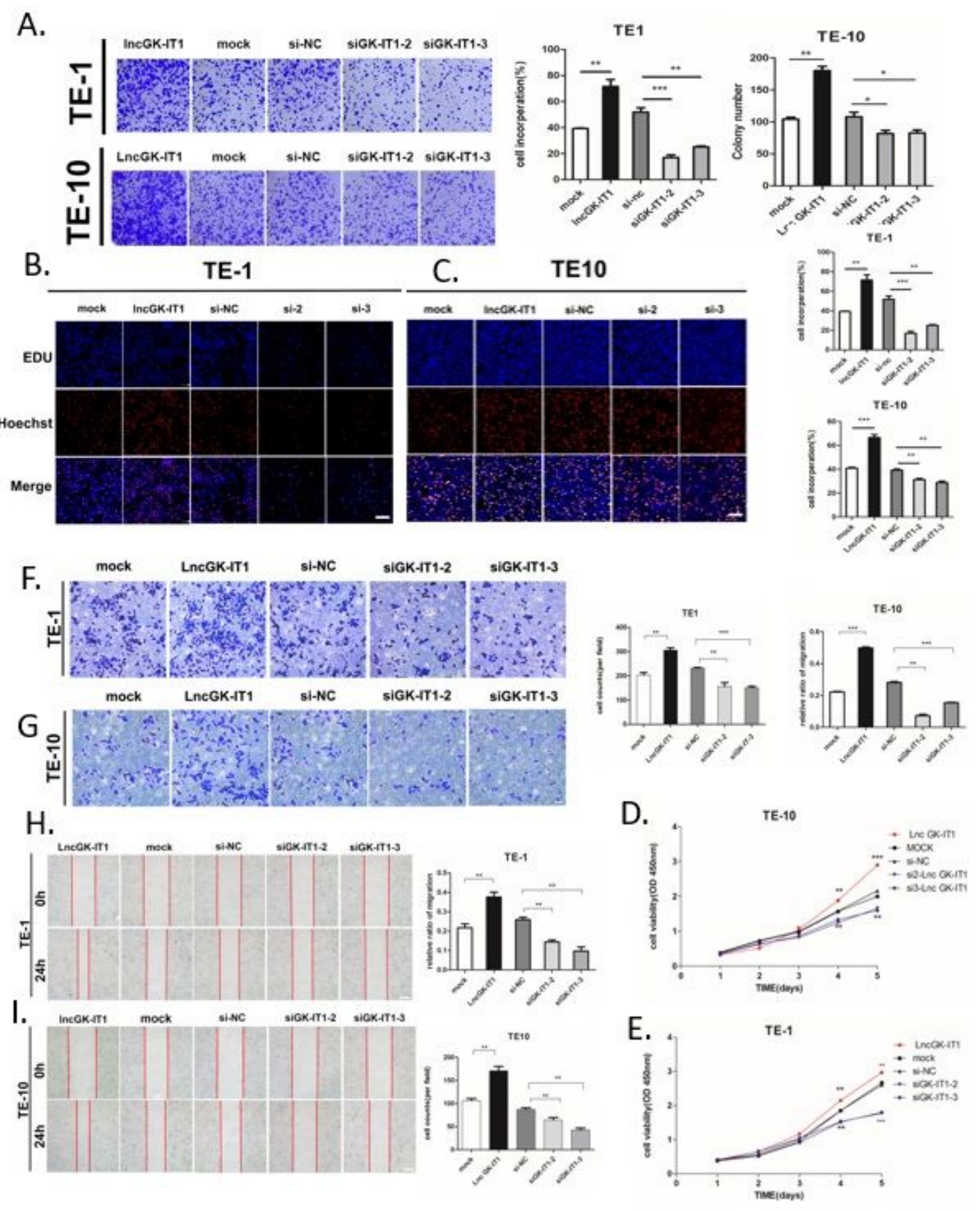

Figure 3

Overexpression and Silencing of GK-IT1 affects proliferation, migration and invasion of ESCC cells a Cell proliferation ability was evaluated by colony formation. b-c EdU assay of ESCC cells was performed to 
evaluate cell proliferation (magnification, $\times 100$, Scale bar, $100 \mu \mathrm{m}$ ). d-e The growth curves of cells were measured by using CCK-8 assay. $\mathrm{f}-\mathrm{g}$ Transwell in vasion assay were used to assess the invasive abilities of ESCC cells (magnification, $\times 100$, Scale bar, $100 \mu \mathrm{m}$ ). h-i The migration abilities of ESCC cells were measured by wound healing (magnification, $\times 50$, Scale bar, $100 \mu \mathrm{m}$ ) assays. The data are presented as the mean $\pm S D$, $* P<0.05$, ** $P<0.01$, *** $P<0.001$
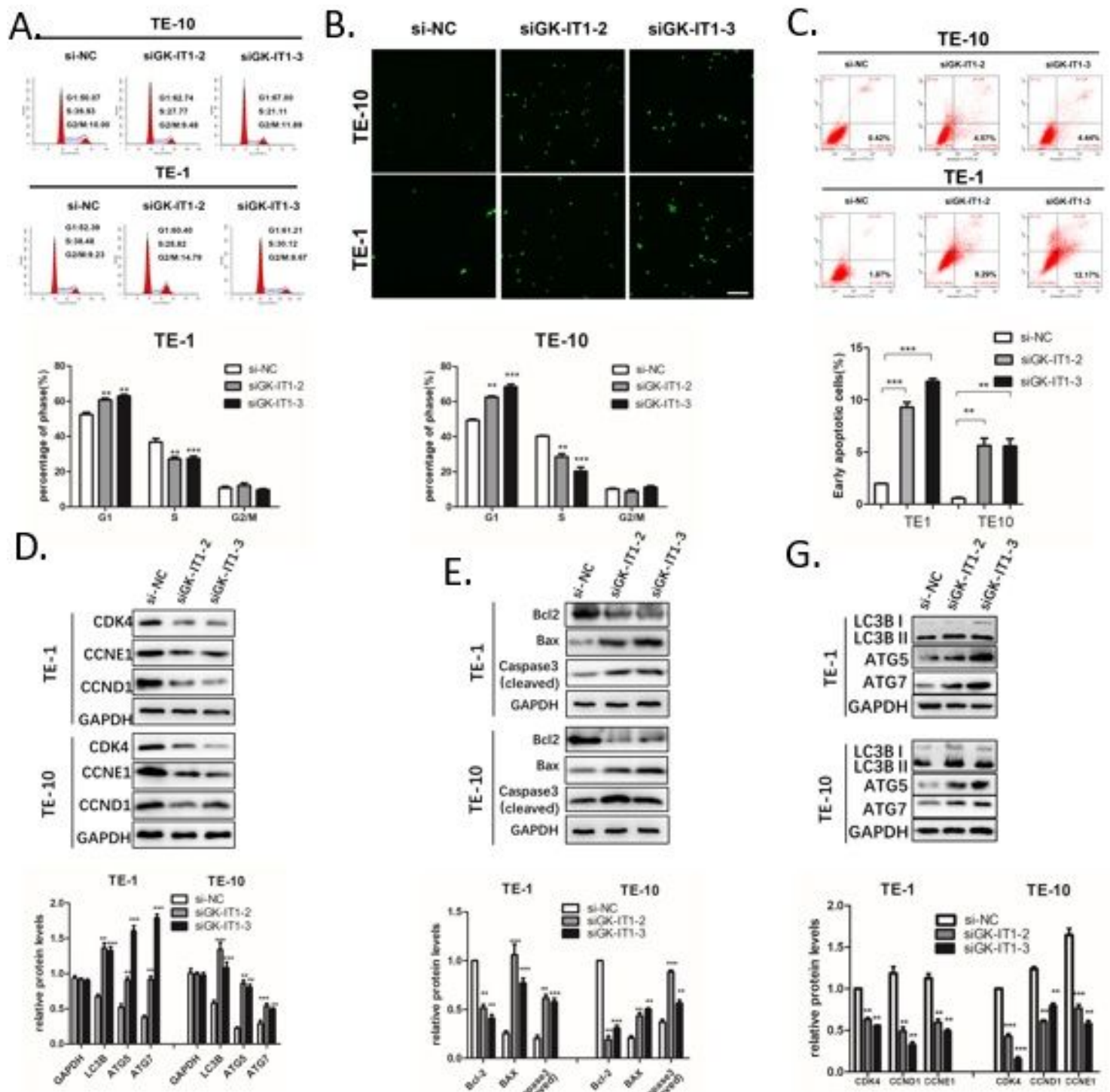

G.

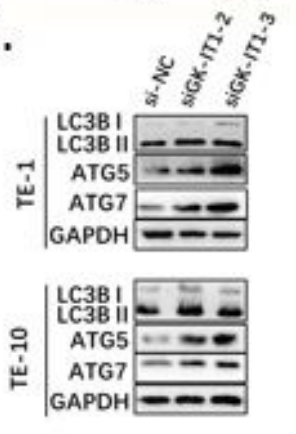

F.
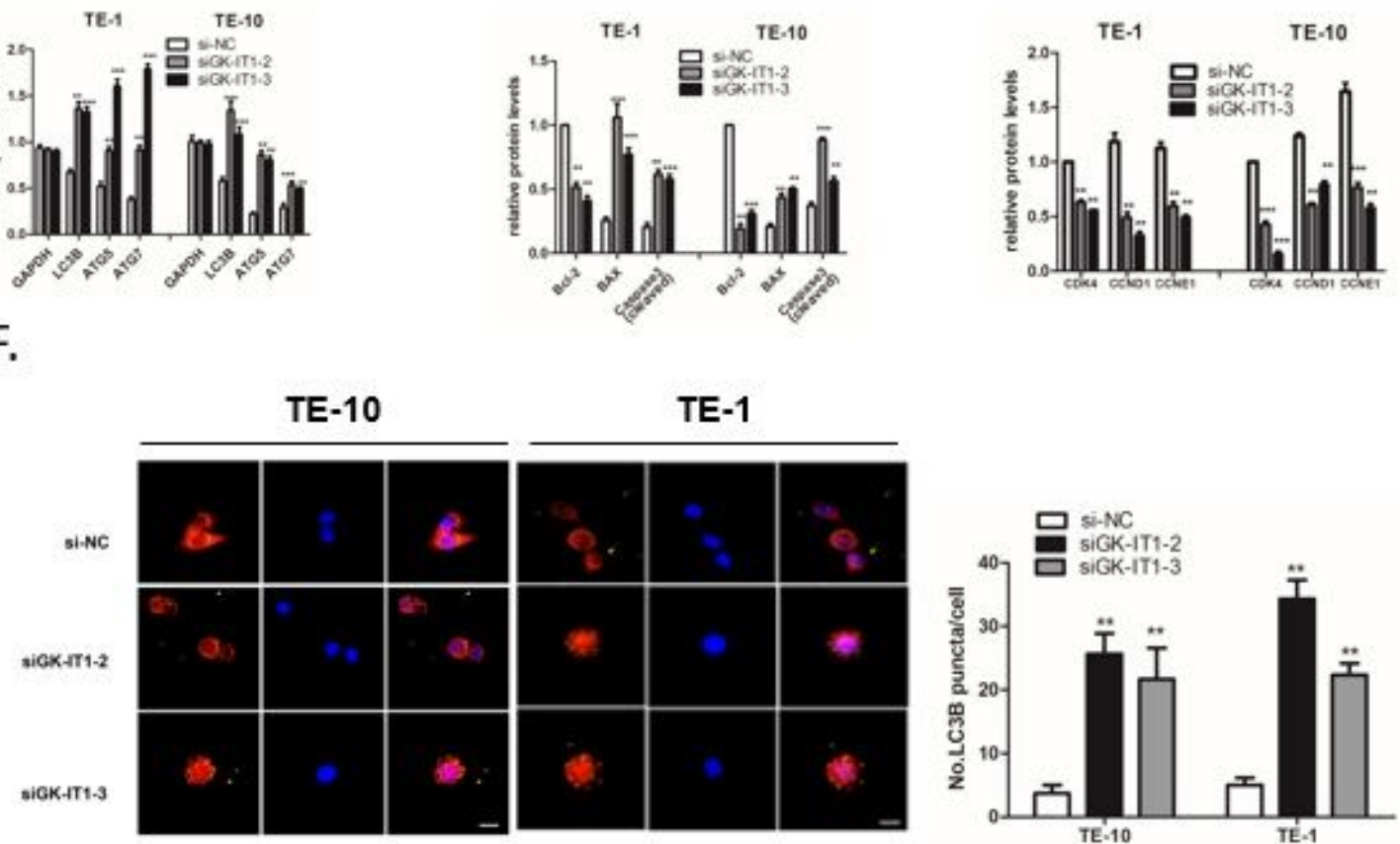

TE-1
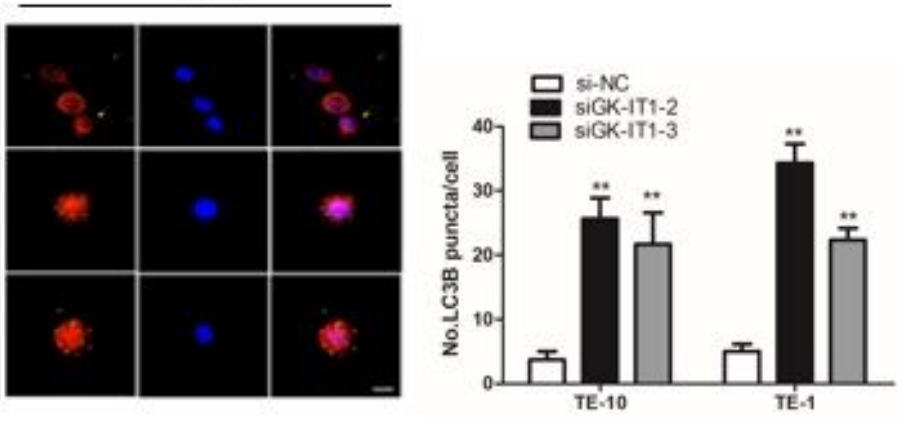

Figure 4 
Knockdown of GK-IT1 induces cell cycle arrest, apoptosis and autophagy of ESCC cells a Cell cycle analysis was performed using flow cytometry in TE-1,TE-10 cells transfected with si-GK-IT1.b Apoptotic ESCC cells were observed with TUNEL method (magnification, $\times 100$, scale bar, $100 \mu \mathrm{m}$ ). c Apoptosis rate was detected using flow cytometry after knockdown GK-IT1. $d$ Western blot was conducted to determine the expression of the cell cycle-related proteins in ESCC cells after transfection with si-GK-IT1. e Western blot was conducted to determine the expression of the cell apoptosis-related proteins in ESCC cells after transfection with si-GK-IT1. $f$ The endogenous LC-3B puncta formation was assessed by IF analysis and the total number of LC3B puncta per cell was counted (magnification, $\times 400$, Scale bar, $20 \mu \mathrm{m}$ ). $\mathrm{g}$ The levels of autophagy-related proteins were detected by western blot. The data are presented as the mean \pm $\mathrm{SD},{ }^{\star} \mathrm{P}<0.05,{ }^{* *} \mathrm{P}<0.01,{ }^{* \star *} \mathrm{P}<0.001$. 
A.

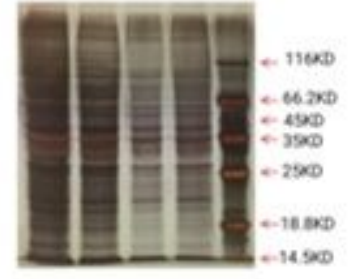

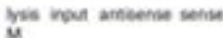

C.

\begin{tabular}{|c|c|c|c|c|}
\hline Protein Names & - Unuses:-1 & Sequence Coveragel:- & Unique PepSeq & - Unique Peptid = \\
\hline UQCRC1 & 2 & 2500000037 & LCTSATESEVAR & 4 \\
\hline CTSO & 203 & 2.426999994 & VSTLPAITLK & \\
\hline ANXA11 & 2 & 2376000024 & DAQELLYAGENR & \\
\hline CDKI & 251 & 3.703999892 & IGEGTYGWYK & \\
\hline EIF2 & 28 & 2331000008 & GVTIKPTVDDD & \\
\hline MAPK1 & 208 & 2222000062 & ICDFGLAR & \\
\hline Cuc1 & 402. & 7.054000348 & YISNAYARIGNCPFSQR & \\
\hline PABPCI & 2 & 1.415000018 & SGVGNFIK & \\
\hline PEPP1 & 142 & 4.278000072 & LYEQLSGK & \\
\hline
\end{tabular}

G.

F.

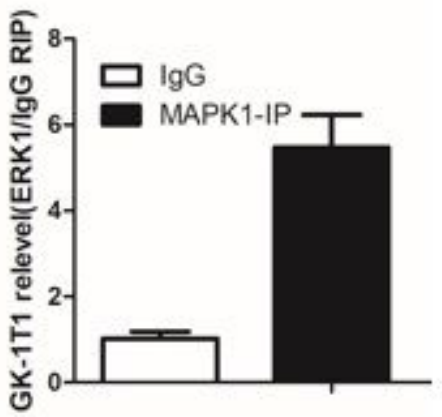

$\mathrm{H}$.

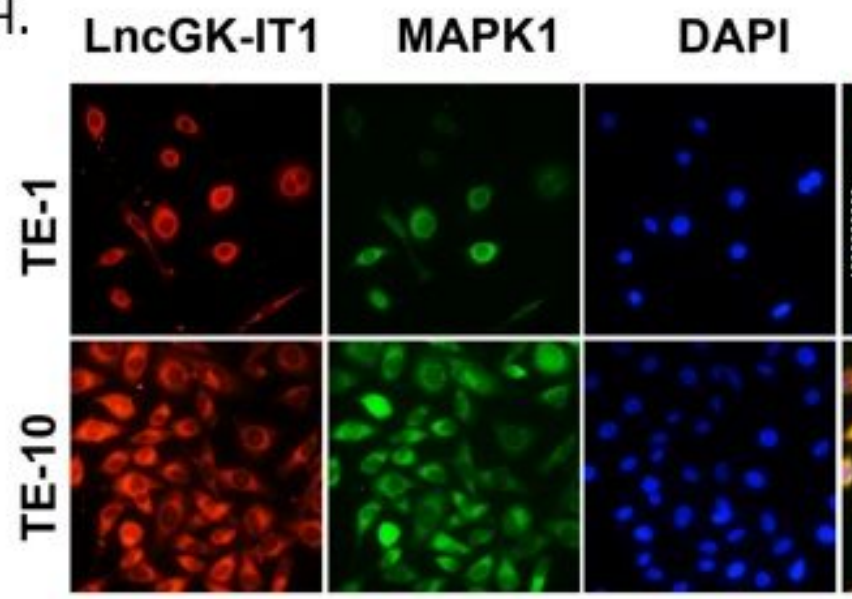

D.

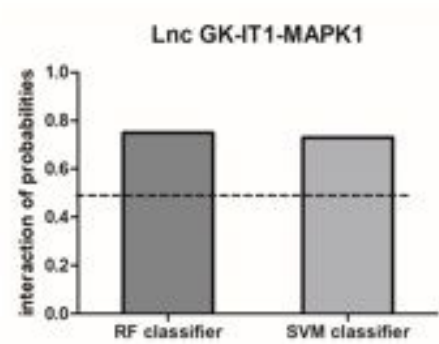

E.

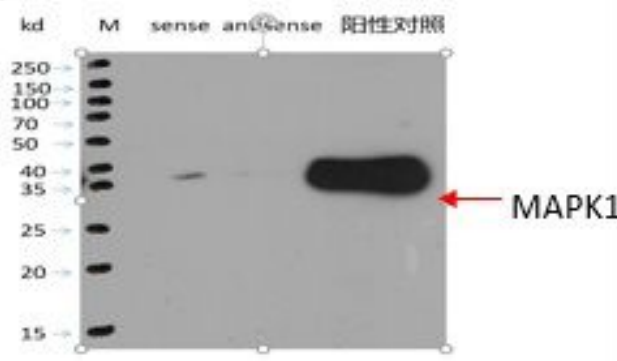

kd $M$ IP IgG Input

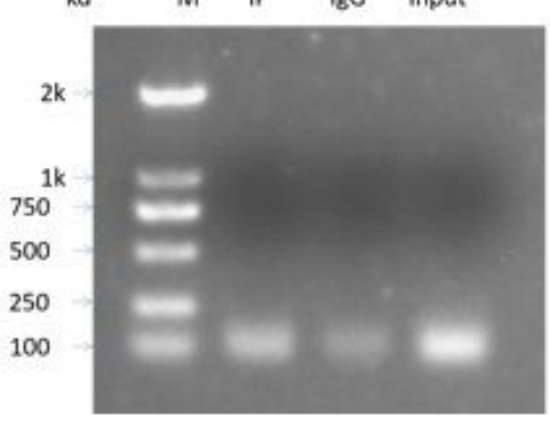

Merge

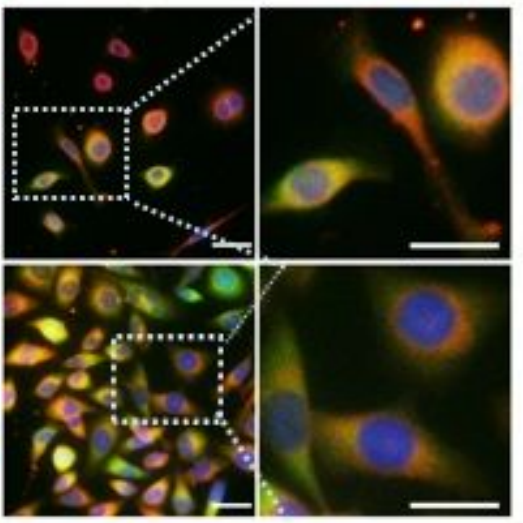

\section{Figure 5}

GK-IT1 interacts with MAPK1 a Bioinformatics analysis of the possibility of interaction between GK-IT1 and MAPK1. b Silver staining of biotinylated GK-IT1-associated proteins after RNA pull-down. Antisense was used as a RNA control. c GK-IT1-bound proteins with cancer relation identified by mass spectrometry. $d$ Western blotting of MAPK1 following pull-down of GK-IT1 or negative RNA control. $f$ The co-localization of GK-IT1 and MAPK1 was observed in ESCC cells (magnification, $\times 400$, Scale bar, $50 \mu \mathrm{m}$ ) by FISH and IF assay. $g$ Cell lysates were immunoprecipitated with MAPK1 or IgG antibody. Expression levels of GK- 
IT1 in either immunoprecipitates or cell lysates (input) of ESCC cells were measured by qRT-PCR (mean \pm s.d. , $n=3$ ). $h$ Expression levels of GK-IT1 in either immunoprecipitates or cell lysates (input) of ESCC cells were measured by Agarose gel electrophoresis.

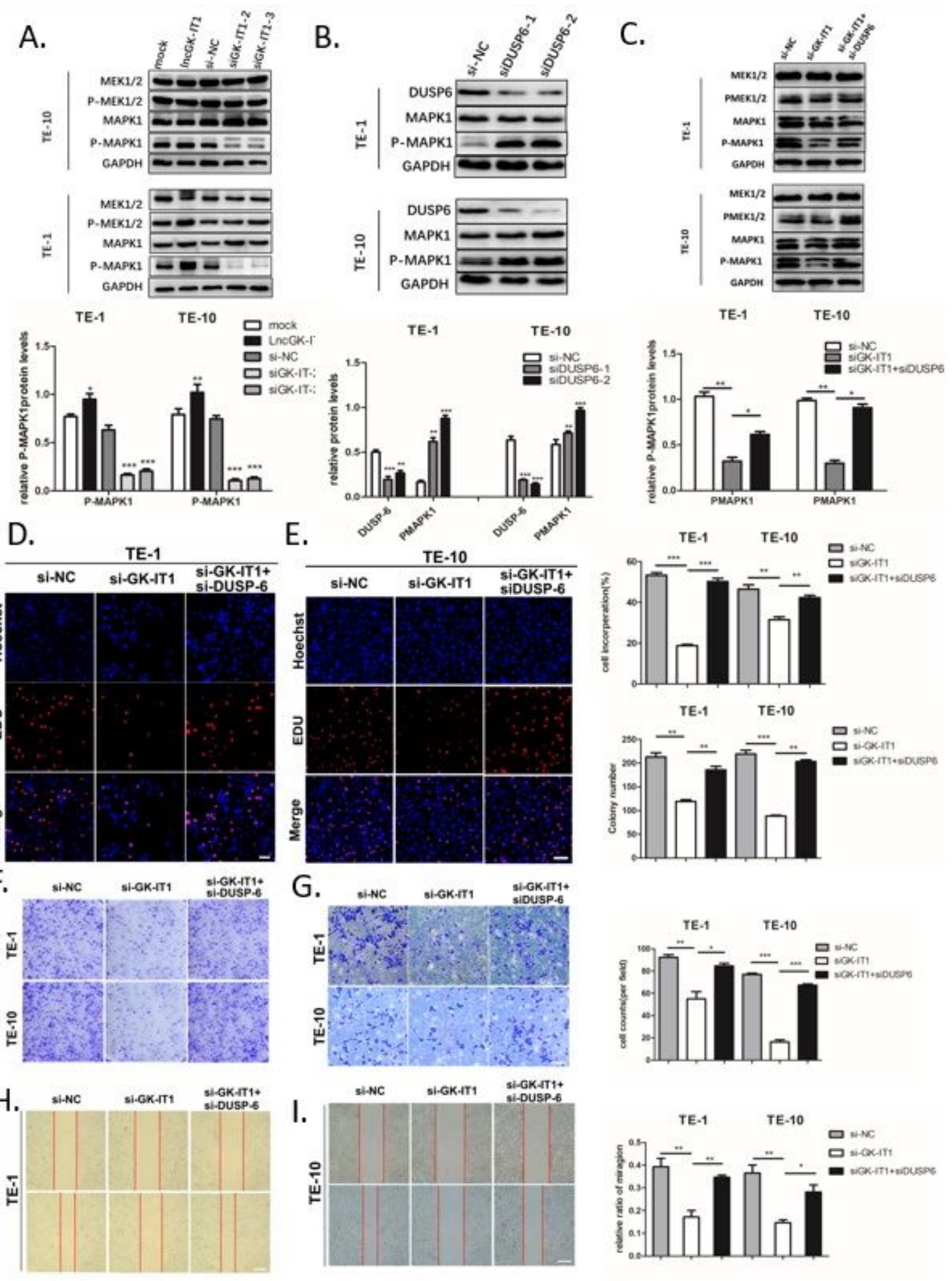

Figure 6

GK-IT1 promotes ESCC progression via activation of ERK/MAPK signalling pathways a Western blot was conducted to determine the expression of the ERK/MAPK1pathways related-proteins in ESCC cells after 
transfection with si-GK-IT1 and overexpressing GK-IT1. b Knockdown DUSP-6 increased expression of ERK/MAPK1 related proteins in ESCC cells by western blotting. $c$ The relative protein levels of ERK/MAPK pathway-related molecules were measured in ESCC cells after transfection or co-transfection with indicated si-NC, siRNAs by western blot. d-f The cell viability of ESCC cells were detected after transfection or co-transfection with indicated si-NC, si-RNAs by EdU (magnification, $\times 200$, Scale bar, 100 $\mu \mathrm{m}$ ) and colony formation assays. $\mathrm{g}-\mathrm{i}$ The invasive and migrative abilities of ESCC cells transfected or cotransfected with indicated si-NC, siRNAs, were monitored by transwell invasion (magnification, $\times 100$, Scale bar, $100 \mu \mathrm{m}$ ), and wound healing (magnification, $\times 50$, Scale bar, $100 \mu \mathrm{m}$ ) assays, respectively. The data are presented as the mean $\pm S D,{ }^{*}<0.05$, ${ }^{\star *} P<0.01$, ${ }^{\star \star *} P<0.001$. 


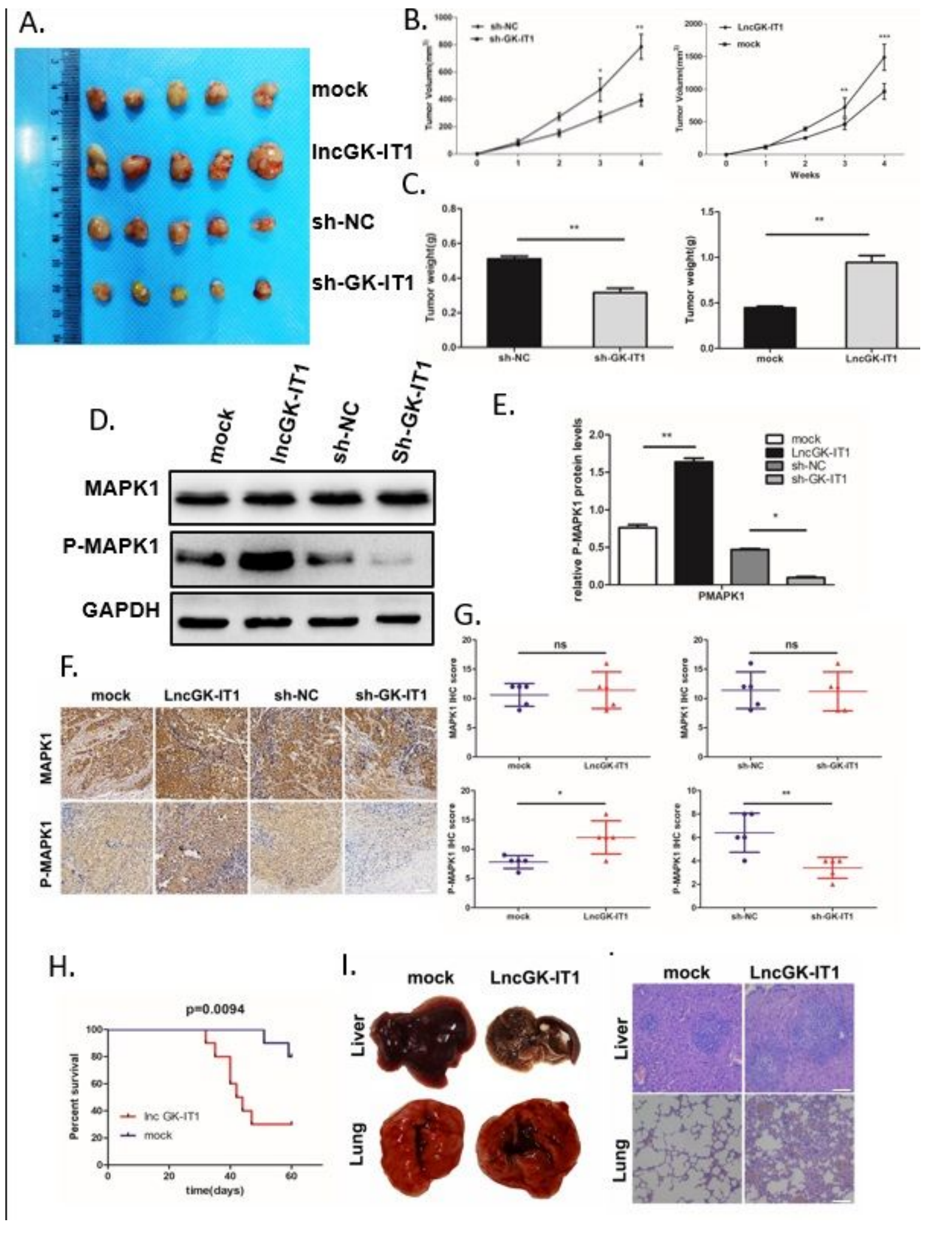

Figure 7

GK-IT1 promotes oncogenesis and metastasis of ESCC in vivo a The representative images of xenograft tumour in each group were displayed $(n=5)$. b tumours volume was measured once a week to plot the growth curve. c Tumour weight was measured. d-e Western blot analysis was conducted to detect the protein level of MAPK1 and P-MAPK1 in xenograft tumours. $\mathrm{f}-\mathrm{g}$ The protein expression levels of MAPK1 and P-MAPK1 in xenograft were verified by immunohistochemical experiment. $\mathrm{h}$ The survival curve of the 
nude mice injected with TE-10 cells transfected with GK-IT1 overexpressing or mock vector was drawn using Kaplan-Meier method. i-j H\&E staining of the lungs (magnification, $\times 200$, Scale bar, $100 \mu \mathrm{m}$ ) and livers (magnification, $\times 200$, Scale bar, $100 \mu \mathrm{m}$ ) were showed.

\section{Supplementary Files}

This is a list of supplementary files associated with this preprint. Click to download.

- GraphicalAbstract.jpg

- additionalfiles.docx 